\title{
Akademisierung in der Diskussion
}

\section{Für eine Kooperation zwischen Hochschule, Weiterbildung und Unternehmen}

\author{
MIRIAM SCHÄFER \\ MICHAEL KRIEGEL, \\ SEBASTIAN WIESCHOWSKI \\ Michael Kriegel ist wissenschaftli- \\ cher Mitarbeiter an der Fachhoch- \\ schule der Diakonie in Bielefeld \\ und Leiter des Forschungsprojekts \\ »BEST-WSG - Berufsintegrierte \\ Studiengänge zur Weiterqualifizie- \\ rung im Sozial- und Gesundheitswe- \\ sen «. Im Rahmen des Wettbewerbs \\ "Aufstieg durch Bildung" des \\ Bundesministeriums für Bildung \\ und Forschung soll die Durchlässig- \\ keit von beruflichen hin zu akade- \\ mischen Abschlüssen im Sozial- und \\ Gesundheitswesen gesteigert wer- \\ den - mit neuartigen Studienmodel- \\ len und innovativen Begleitstruktu- \\ ren. Miriam Schäfer und Sebastian \\ Wieschowski sind wissenschaftliche \\ Mitarbeiter in diesem Projekt. \\ www.offene-fh.de
}

\author{
Die "Akademisierung der Pflege" wird immer \\ wieder gefordert. Viele Praktiker sehen diesen \\ Wunsch eher kritisch. Eine guter Weg könnte \\ die abgestimmte Zusammenarbeit zwischen \\ Hochschulen, Weiterbildungseinrichtungen \\ und Anstellungsträgern sein.
}

In Deutschland wird händeringend qualifiziertes Personal in der Pflege gesucht. Gleichzeitig tut man sich mit der Aufwertung des Berufsbildes ungemein schwer. Nicht nur die gesellschaftliche Anerkennung bleibt aus, in Fachkreisen selbst wird heftig darum gestritten, welcher Ausbildungsweg beziehungsweise welcher Status in der Pflege benötigt wird. Dies macht sich am deutlichsten an der anhaltenden Diskussion über die hochschulische Qualifikation dieser Branche fest.

Die Schlussfolgerung: Wenn sich selbst für Ausbildungsplätze keine geeigneten Bewerberinnen und Bewerber finden und immer weniger Kolleginnen und Kollegen immer mehr Patienten betreuen, fehlt für die Akademisierung schlicht die Zeit. Zudem sollte, so ist gemeinhin zu hören, die wenige Zeit nicht mit der Aufwertung von bestehenden Berufsbildern verschwendet, sondern für die Suche nach neuen Zielgruppen genutzt werden - Hauptschüler, Querund Wiedereinsteiger, Menschen aus »bildungsfernen Schichten" oder osteuropäischen Ländern.

Hierbei wird offenkundig verkannt, dass die Aufgaben und großen Anforderungen in einer immer älter werdenden Bevölkerung mit chronischen und komplexen gesundheitlichen Versorgungsbedarfen stetig wachsen und zukünftig wohl immer mehr Pflegekompetenzen auf wissenschaftlicher Basis benötigt werden. Unter anderem hat dies der Wissenschaftsrat im Sommer 2012 mit seiner Empfehlung zur hochschulischen
Qualifikation für das Gesundheitswesen deutlich gemacht.

Doch wie kann hochschulische Bildung für die pflegenden Berufe gelingen? Welche Rahmenbedingungen sind dafür erforderlich? Welche Art der Akademisierung wird von der Praxis überhaupt gewünscht? Diesen Fragen ist die Fachhochschule der Diakonie in Bielefeld im Rahmen ihres vom Bundesministerium für Bildung und Forschung geförderten Forschungsprojektes »Aufstieg durch Bildung: Offene Hochschulen« nachgegangen.

Um allerdings in der Komplexität der angedeuteten Akademisierungsdebatte nicht unterzugehen, wurde das Untersuchungsdesign auf bestimmte Aspekte begrenzt und auch in den nachfolgenden Ausführungen konzentrieren wir uns lediglich darauf, wie das Profil der Hochschule, insbesondere im Hinblick auf die Kooperation zwischen Wissenschaft und Praxis, aussehen sollte, um den Ansprüchen in der Sozial- und Gesundheitsbranche sowohl von individueller wie institutioneller Seite gerecht zu werden.

Eine Befragung von insgesamt 29 berufserfahrenen Experten aus Sozialunternehmen, Weiterbildung, Hochschule, Personalvermittlung und Verbandswesen im Zeitraum von März bis Juli 2012 sollte schwerpunktmäßig darüber Aufschluss geben, welche dringenden Bedarfe im Sozial- und Gesundheitswesen bestehen und wie innovative Studiengänge die gewünschten Kompetenzen in Verbindung mit praktischen 
Weiterbildungsangeboten vermitteln werden können. In die spätere Gesamtanalyse wurden die bereits bestehenden Bachelor-Studiengänge »Pflege« sowie "Psychische Gesundheit/Psychiatrische Pflege « der Fachhochschule für Diakonie mit einbezogen.

Im Mittelpunkt der Untersuchung stand also das Zusammenwirken zwischen Wissenschaft und Praxis, zwischen Hochschule, Weiterbildung, sozialen und pflegerischen Einrichtungen. Und aus den bis dato erfolgten Auswertungsergebnissen lassen sich vor allem zwei Erkenntnisse ableiten:

- Hochschule und berufliche Weiterbildung (innerhalb und außerhalb von Unternehmen) behindern sich derzeit eher noch gegenseitig, als dass sie gemeinsame Wege einschlagen.

- Klassische Hochschulausbildung lässt sich offenbar noch immer nicht mit der Struktur moderner Weiterbildungsprogramme verknüpfen. Im Klartext: Es sind in weiten Teilen immer noch zwei völlig getrennte Bildungssysteme. Bei den Expertengesprächen dominierte ein Bild der Hochschule, welches sich durch Wissenschaftlichkeit, die Vermittlung von Theorie, der Freiheit von Forschung und Lehre auszeichnet, aber auch durch Praxisferne, wenig Kunden- und Serviceorientierung und eine gewisse Unflexibilität. Die Weiterbildung nimmt nach Einschätzung der Experten eine fast konträre Position dazu ein - sie gilt als praxisnah, bedarfs- und kundenorientiert, aber auch wenig wissenschaftstheoretisch.

Trotz der noch bestehenden Diskrepanzen sprechen sich die Experten für die Zusammenarbeit zwischen Hochschule und Weiterbildung aus, ja, sie halten sie für geradezu zwingend notwendig, um nachstehende Vorteile in Betracht ziehen zu können:

- Anrechenbarkeit: Sind Weiterbildungsleistungen auf ein Hochschulstudium anrechenbar, erreichen beide Bildungssysteme zusammen eine breitere oder heterogenere Zielgruppen. Weiterbildung gewinnt an Attraktivität, wenn sie anschlussfähig ist und anrechenbar auf ein Hochschulstudium. Durch die Anrechen-

barkeit von Weiterbildung kann wiederum ein Studium für Berufstägige interessant werden, weil sich die Studiendauer möglicherweise verkürzt oder ein niederschwelliger Einstieg möglich wird.

- Gemeinsame Produkte: Entwickeln Hochschule und Weiterbildungsträger (auch in Kooperationen mit Unternehmen) gemeinsame Angebote, wird eine einseitige Orientierung vermieden und eine Anschlussfähigkeit in alle Richtungen geschaffen. Es findet eine Verbindung zwischen individuellen Voraussetzungen und institutionellen Erwartungen statt. Ein gemeinsames Bildungscontrolling im Sinne eines gemeinsamen Kundenbetreuungssystems kann Bedarfe gezielt ermitteln und Angebote gemeinsam schaffen.

- Theorie-Praxis-Transfer: Zentrale Herausforderungen und Zukunftsfragen der Praxis können gemeinsam identifiziert und wissenschaftlich fundiert bearbeitet werden. Hieraus können gemeinsame Angebot generiert werden, die sowohl den Ansprüchen an Praxisnähe als auch an Wissenschaftlichkeit gerecht werden. So profitieren beide Systeme von den Kernkompetenzen des jeweils anderen.

\section{"Irgendwer muss das Pflaster} kleben, dazu brauche ich keinen

\section{Hochschulabschluss»}

- Modularisierung: Modularisierte Studienmodelle bieten individuelle und flexible Qualifizierungsmöglichkeiten. Sie können an unterschiedlichen Lernorten angeboten und durchgeführt werden. So können Teile eines Curriculums, begünstigt durch die modulare Struktur, von Weiterbildungsträgern angeboten werden, so dass eine Nachfrage für beide Seiten garantiert wird.

- Durchlässigkeit: Lebensbegleitendes Lernen und Durchlässigkeit hängen sehr eng miteinander zusammen. Durch eine vertrauensvolle LernortKooperation zwischen Hochschule und Weiterbildung werden durchlässige Bildungswege geschaffen, die eine effizientere Nutzung von Lern- und Lebenszeiten für Weiterbildungs- und Studieninteressierte fördern.

Ebenso eindeutig sprechen sich die befragten Experten für eine engere Verzahnung von Hochschulen und Sozial- und Pflegeeinrichtungen aus und betonen, dass ein kontinuierlicher Theorie-Praxis-Transfer institutionalisiert werden soll. Bei den Befragungen standen daher mögliche Kooperationsformen im Mittelpunkt:

Im Rahmen eines fachbezogenen Austauschs könnte beispielsweise ein regelmäßiges Forum eingerichtet werden, in dem Hochschule und Sozialunternehmen kontinuierlich relevante Praxisthemen und wissenschaftliche Erkenntnisse erörtern. Zudem sollten die Anforderungsprofile und Bedarfe der Unternehmen bei der Curriculumsentwicklung berücksichtigt werden. Bei den Expertengesprächen wird unter anderem die Einrichtung einer Informationsbörse vorgeschlagen, in der konkrete Anfragen und Angebote eingebracht und abgefragt werden können. Denkbar wäre auch die Identifizierung von Zukunftstrends in der Sozial- und Gesundheitswirtschaft, beispielsweise durch sogenannte Themenscouts, so- 
von kompetenzorientierten Prüf- und Einstellungsverfahren. Zudem müssten Studium und Weiterbildung in interne Unternehmensprozesse integriert werden. Hier werden insbesondere duale Studienmodelle positiv hervorgehoben.

\section{Die Praxis in das Studium integrieren}

In den Expertengesprächen wurde insbesondere die Notwendigkeit der Integration von Praxisanteilen im Studium genannt. Dies ist aus Sicht der Experten möglich beispielsweise über Praktika, Praxissemester, Projekte (Lernen an realen Fällen aus der Praxis, problemorientiertes Lernen), die Mitarbeitergewinnung durch Praxiskontakte im Studium, Aufbau von Modellprogrammen (im Unternehmen lernen) sowie ein berufsintegriertes Studium.

Aus den genannten Bedarfen ergeben sich, wie die Expertengespräche deutlich machen, für das Zusammenwirken von Hochschule und Unternehmen konkrete Kompetenzverteilungen: Die Hochschule könnte demnach InhouseSeminare in Einrichtungen und Unternehmen anbieten, Forschungs- und Evaluationsaufträge aus Sozial- und Pflegeunternehmen übernehmen und beratend in Betrieben tätig werden. Im Gegenzug beteiligen sich die Einrichtungen und Unternehmen an der Themenstellung und Vergabe von Abschlussarbeiten. Zudem könnten Professoren in die unternehmerische Praxis eingebunden werden und Praktiker aus den Unternehmen am wissenschaftlichen Diskurs teilnehmen.

Aus den Expertenmeinungen lassen sich eine Reihe von konkreten Maßnahmen ableiten. Im Zuge der Curriculumsentwicklung scheint es ratsam, soziale und pflegende Dienstleistungsanbieter von vornherein zu beteiligen. Eine mögliche Form ist die Bildung von Kompetenzgruppen für die jeweiligen Fachgebiete, die sowohl aus aus Vertreterinnen und Vertretern von Unternehmen, Hochschule und Weiterbildungsträgern besetzt sein sollen. Durch die gemeinsame Entwicklung wird somit nicht nur ein Produkt geschaffen, das von allen Beteiligten mitgetragen wird, sondern auch die Chance hat, von Anfang an in den Einrichtungen und Unternehmen, beispielsweise im Sinne der Nachwuchsförderung, etabliert zu sein. Durch die Zusammenarbeit mit den Weiterbildungsträgern können die Vorteile beider Systeme ausgeschöpft werden und bereits in der Entwicklung Aufgaben oder Module an die Weiterbildungsträger ausgelagert werden, die dort bedingt durch die Praxisnähe besser aufgehoben sind.

Durch die gemeinsame Entwicklung werden die Praxisanteile für die Pflege identifiziert: Was kann sinnvollerweise vor Ort, am Arbeitsplatz im täglichen Pflegebetrieb, im Unternehmen selbst gelernt werden? Auf dieser Grundlage können beispielsweise Konzepte für integrierte Care-Management-Modelle oder Case-Studies, Praxismodule sowie Trainings-on-the-job entwickelt und direkt mit den Einrichtungsverantwortlichen abgestimmt werden. Die Vergabe von Themen für die Abschlussarbeiten könnte im Rahmen einer onlinegestützten Themenbörse umgesetzt werden, ebenso eine Jobbörse für die Absolventen. Evaluations- und Forschungsaufträge der Unternehmen sollten wenn möglich ins Curriculum eingebunden sein: In Forschungsmodulen könnten Studierendengruppen dies in Begleitung eines Professors übernehmen.

Ein weiterer Vorteil in der engen Verzahnung von Hochschule und Sozialunternehmen bei der Akademisierung der Pflege liegt darin, dass der Effekt des Studiums sichtbar wird und somit das Unternehmen unmittelbar profitieren kann. Dies könnte dazu beitragen, dass die Bereitschaft der Unternehmen steigt, sich an der Akademisierung stärker zu beteiligen.

In einer engeren Kooperation von Hochschule und Unternehmen liegen also viele Vorteile für beide Seiten. Kontrovers diskutiert wird von den Experten die Frage, wie weit Hochschule unmittelbar für die soziale und pflegerische Praxis ausbilden soll oder was im Sinne der Freiheit der Lehre auch noch zweckfrei bleiben soll und darf. Ein Experte eines Sozialunternehmen hat dies in einem der Interviews folgendermaßen auf den Punkt gebracht: »Es muss darauf geachtet werden, dass die Eigenständigkeit des Studiengeschehens gewährleistet, die sich bewusst abhebt von einer direkten Eins-zu-Eins Entsprechung von Kompetenzerwartungen aus der Praxis."

Die Bedarfserhebung hat abseits der konkreten Hinweise zur Gestaltung der
Zusammenarbeit zwischen Hochschule, Sozialunternehmen und Weiterbildungsträgern eines deutlich gemacht: Die Akademisierung der Pflege wird als Ausbildungsoption dringend benötigt. Sie eignet sich aus Sicht der Befragten insbesondere für Pflegedienstleitung, Hausleitung, Pflegedirektoren und Primary Nurses. Allerdings ist eine flächendeckende Akademisierung der Pflege nach Einschätzung der Befragten unrealistisch: "Ich glaube auch nicht, dass wir am Ende des Tages in der Krankenpflege nur Hochschülerinnen und Hochschüler haben, weil irgendeiner muss das Pflaster kleben und dazu brauche ich keinen Hochschulabschluss ", heißt es in einer Einschätzung eines Befragten aus dem Pflegebereich. Hier wird ein verstärkter Einsatz von Hilfskräften, die dann von den Fachkräften angeleitet werden müssen, wahrscheinlich.

Last, but not least: Von einer Akademisierung der Pflegeberufe profitieren alle Seiten - die Pflegenden erhalten den nötigen Hintergrund, um die vielfältige Herausforderungen ihres Berufs souverän zu meistern, während die Pflegeunternehmen ihre Organisation fit für die Zukunft machen und durch die akademische Anbindung aktuelle Impulse erhalten, die sofort in den Pflegealltag übertragen werden.

\section{Konkurrenzfähig werden - und bleiben}

Mit ausbildungs- und berufsbegleitenden Pflegestudiengängen wird die Wissenschaft an die Basis gebracht, um die Patientenbetreuung langfristig zu optimieren. Und nicht zuletzt bleiben Pflegedienstanbieter, die auf eine akademische Qualifizierung setzen, im internationalen Bewerbermarkt konkurrenzfähig: Anderswo gehört der akademische Grad eines »Bachelor of Nursing « längst zum Standard - in Deutschland ist er noch ein Fremdwort. Dabei sind im europäischen Ausland auch Pflegerinnen und Pfleger mit einem Bachelorabschluss weiterhin im täglichen Dienst und dem direkten Kontakt mit den Menschen verwurzelt. 